\title{
A generalization of the principal ideal theorem
}

\author{
By Fumiyuki TeradA
}

(Received Nov. 25, 1955)

The purpose of this paper is to give a cohomology-theoretical description of a generalized principal ideal theorem. The definitions and the notations in this paper are borrowed from C. Chevalley's lecture notes at Nagoya University [1].

1. Let $G$ be a finite group, and $S$ be an automorphism of the group $G$. The image of an element $\sigma \in G$ by $S$ will be denoted by $S(\sigma)$. Let $H$ be the invariant subgroup of $G$, which is generated by all the elements $S(\sigma) \sigma^{-1}, \sigma \tau \sigma^{-1} \tau^{-1}(\sigma, \tau G)$. Then $H$ is an $S$-invariant subgroup of $G$, and $G / H$ is abelian.

Let $A$ be a $G$-module. We shall denote a submodule of $A$ which is generated by all the elements $(1-\sigma) a(\sigma \in G, a \in A)$ by $I_{G} A$. Especially, if $A$ is the group $\operatorname{ring} Z(G)$ over the integral domain $Z$ of all rational integers, the submodule $I_{G} Z(G)$ will be denoted by $I_{G}$. We shall use analogous symbols concerning subgroups of $G$.

2. We shall consider, in this section, certain mappings of the cohomology groups of $G$.

Let $x, y \in I_{G}$, and $n \in Z$. Then, $x \otimes y \otimes n \rightarrow x \otimes n y$ defines an isomorphism $\psi_{G}: H^{-2}(G, Z) \rightarrow H^{-1}\left(G, I_{G}\right)$. We have also an isomorphism $\psi_{H}: H^{-2}(H, Z) \rightarrow H^{-1}\left(H, I_{H}\right)$.

Let $A$ be a $G$-module, and $\tau \in G, a(\tau) \in A$ such that $\sum a(\tau)=0$. Then, $\sum \tau \otimes a(\tau) \rightarrow a(e)$, where $e$ is the unit element of $G$, induces an isomorphism $H^{-1}(G, A) \rightarrow A^{G \rightarrow 0} / I_{G} A$. Especially, if $A=I_{G}$, we have an isomorphism $\varphi_{G}: H^{-1}\left(G, I_{G}\right) \rightarrow I_{G} / I_{G} I_{G}$, and also, $\varphi_{H}: H^{-1}\left(H, I_{G}\right) \rightarrow I_{G}^{H \rightarrow 0} / I_{H} I_{G}$.

We have also an isomorphism $\Phi: H^{-1}\left(H, I_{H}\right) \rightarrow H^{-1}\left(H, I_{G}\right)$ (cf. [1] Theorem 7.1).

Let $j_{-r}$ be the injection mapping $H^{-r}\left(H, I_{G}\right) \rightarrow H^{-r}\left(G, I_{G}\right), \boldsymbol{r}=1,2$. Then, $\boldsymbol{\varphi}_{G} \boldsymbol{j}_{-1} \boldsymbol{\varphi}_{H}^{-1}$ maps $I_{G}^{H \rightarrow 0} / I_{H} I_{G}$ into $I_{G} / I_{G} I_{G}$, and the kernel is the subgroup $\left(I_{G^{\prime}}, I_{H} I_{G}\right) / I_{H} I_{G}$ of the group $I_{G}^{H \rightarrow 0} / I_{H} I_{G}$, where $G^{\prime}$ is the commutatorsubgroup of $G$.

The ideal $I_{G}$ of $Z(G)$ is generated by all the elements $1-\sigma, \sigma \in G$, and each element of $I_{G}$ is described as $\sum a(\sigma)(1-\sigma)$, where $a(\sigma) \in Z$. 
Then, $\sum a(\sigma)(1-\sigma) \rightarrow \sum a(\sigma)\left(1-\sigma^{-1} S(\sigma)\right)$ defines a homomorphism $S^{*}$ of the module $I_{G}$ into $I_{H^{*}}$ And when we consider the ideal $I_{H}$ modulo $\left(I_{G^{\prime}}, I_{H} I_{G}\right), S^{*}$ induces a $G$-homomorphism of the $G$-module $I_{G}$ into $I_{H} /\left(I_{G^{\prime}}, I_{H} I_{G}\right)$. The kernel of this homomorphism contains $I_{G} I_{G}$. Thus we have a $G$-homomorphism $S_{1}^{*}: I_{G} / I_{G} I_{G} \rightarrow I_{G}^{H \rightarrow 0} /\left(I_{H} I_{G}, I_{G^{\prime}}\right)$, and combining with $\psi, \varphi$, we may define a homomorphism $S_{2}^{*}: H^{-2}(G, Z) \rightarrow H^{-2}(H, Z) /$ (kernel of $j_{-2}$ ). More precisely, $S_{2}^{*}=\boldsymbol{\psi}_{H}^{-1} \bullet \Phi^{-1} \cdot \boldsymbol{\varphi}_{H}^{-1} S_{1}^{*} \boldsymbol{\varphi}_{G} \boldsymbol{\psi}_{G}$, and if $\sum \boldsymbol{\sigma} \otimes$ $a(\sigma) \in\left(I_{G} \otimes I_{G}\right)^{G}, a(\sigma) \in I_{G}, \sum a(\sigma)=0$, then $S_{2}^{*}$ maps $\sum_{\sigma \in G} \sigma \otimes a(\sigma)$ to $\sum_{h \in G} h \otimes h S^{*}(a(e)) \in\left(I_{H} \otimes I_{H}\right)^{H}$.

Moreover, we shall consider the restriction mapping. We shall describe it in details (cf. [1], Chap. 7). Let $\sigma_{1}=1, \sigma_{2}, \cdots, \sigma_{m}$ be representatives of the quotient group $G / H$, and $X$ be a submodule of the module $I_{G}$ which is generated by $\sigma_{i}-1(i=1, \cdots, m)$. Then $I_{G}=$ $I_{H}+U$ (direct), where $U=\sum_{h \in H} h X$. Let $\alpha$ be the mapping of $I_{G}$ onto $I_{H}$ which maps $U$ upon 0 and maps $I_{H}$ identically. Then the mapping $\alpha \otimes \alpha: I_{G} \otimes I_{G} \rightarrow I_{H} \otimes I_{H}$ defines the restriction mapping $r_{2}: H^{-2}(G, Z) \rightarrow$ $H^{-2}(H, Z)$. Also, $\alpha \otimes 1: I_{G} \otimes I_{G} \rightarrow I_{H} \otimes I_{G}$ defines the restriction mapping $r_{1}: H^{-1}\left(G, I_{G}\right) \rightarrow H^{-1}\left(H, I_{G}\right)$, and we have a commutativity relation $\boldsymbol{r}_{1} \cdot \psi_{G}=\Phi \cdot \psi_{H} \cdot \boldsymbol{r}_{2} \cdot \quad$ Moreover, $\quad \boldsymbol{r}=\boldsymbol{\varphi}_{H} \boldsymbol{r}_{1} \boldsymbol{\varphi}_{G}^{-1} \quad$ is a mapping $I_{G} / I_{G} I_{G} \rightarrow$ $I_{G \rightarrow 0}^{H \rightarrow} I_{H} I_{G}$ and by the definition of $\alpha, r$ is a mapping which maps $x \in I_{G}$ to $\sum_{i=1}^{m} \sigma_{i} x \in I_{G}^{H \rightarrow 0}$.

Finally, we shall describe these mappings in the following diagrams, which are commutative.
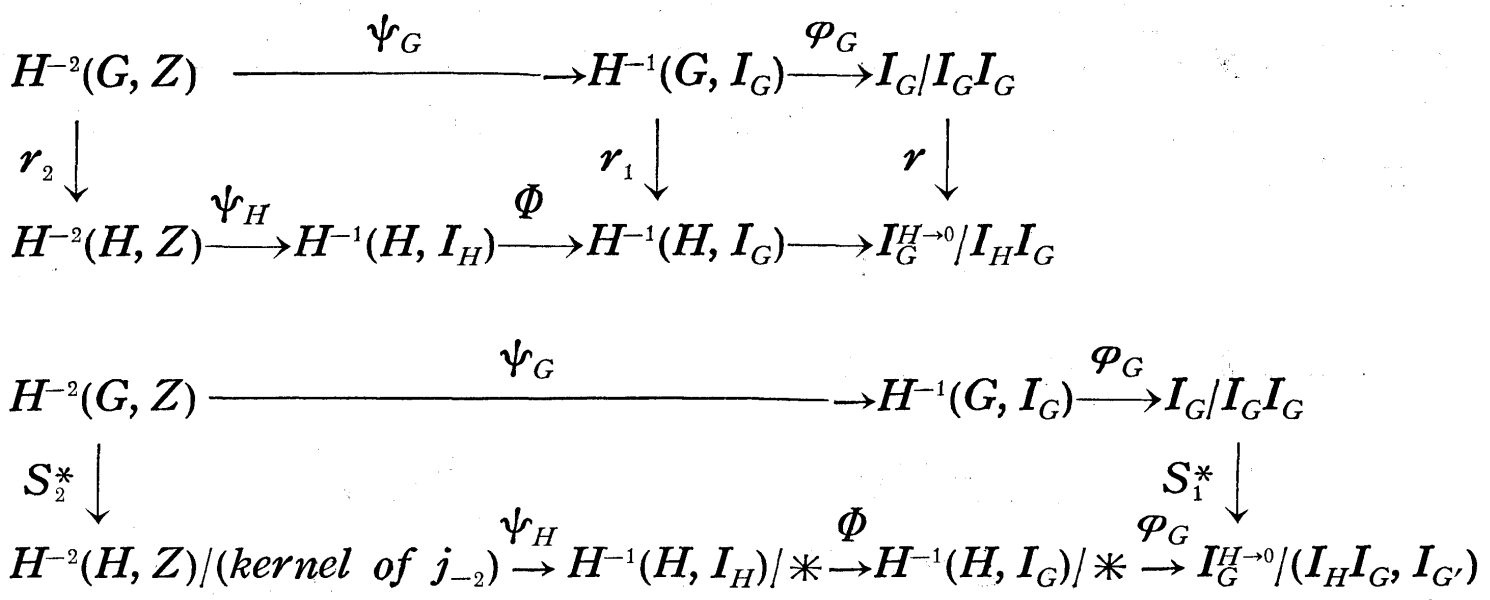
3. In this section, we shall prove the following proposition.

PROPOSITION. Kernel of $S_{1}^{*} \subset$ kernel of $r$.

Let $\tau_{1}, \cdots, \tau_{m}$ be representatives of generators of the abelian group $G / H$, where we may assume that these elements generate the group $G$. This is accomplished by adding to them certain elements of $H$. Let $e_{1}, \cdots, e_{m}$ be the order of $\tau_{1}, \cdots, \tau_{m} \bmod H$. Then, $a_{i}=1-\tau_{i}(i=1, \cdots, m)$ form an ideal base of $I_{G}$.

Let $M$ be the direct sum $\sum_{i=0}^{l-1} Z(G) \bar{S}^{i}$, where $\bar{S}$ is a symbol such that $\bar{S}^{\prime}=1$ and $l$ is the order of the automorphism $S$. If we define $\bar{S}\left(\sigma \bar{S}^{i}\right)=S^{-1}(\sigma) \bar{S}^{i+1}$, then $M$ will have the structure of a ring. Let $I_{M}$ be the ideal of $M$ generated by all the elements $1-\xi, \xi \in M$. Then $a_{0}=1-\bar{S}$ and $a_{i}(i=1, \cdots, m)$ form an ideal base of $I_{M}$.

Let $\bar{I}_{G}=M I_{G}, \bar{I}_{H}=M I_{H}$, and $\bar{I}_{G^{\prime}}=M I_{G^{\prime}}$. In this section, we shall consider the module $\bar{I}_{G}$ modulo $\bar{I}_{H} I_{M}$, where $\bar{I}_{H} I_{M}$ is an ideal of $M$ generated by all the elements $(1-h)(1-m), h \in H, m \in M$. Then, $\bar{I}_{H} I_{M} \subset \bar{I}_{H}$, and $\bar{I}_{H}$ is generated by the following elements (1), (2) modulo $\bar{I}_{H} I_{M}$.

$$
\begin{array}{ll}
\left(1-\tau_{i}\right) a_{j}-\left(1-\tau_{j}\right) a_{i} & (i, j=1, \cdots, m) \\
(1-\bar{S}) a_{i}-\left(1-\tau_{i}\right) a_{0} & (i=1, \cdots, m)
\end{array}
$$

And $\left(\bar{I}_{G^{\prime}}, \bar{I}_{H} I_{M}\right)$ is generated by elements (1) modulo $\overline{\boldsymbol{I}}_{H} \boldsymbol{I}_{M}$. It is shown easily that $x y a_{j} \equiv y x a_{j} \bmod \bar{I}_{H} I_{M},(j=0,1, \cdots, m)$, where $x, y \in G$ or $x=\bar{S}$ or $y=\bar{S}$. In other words, we can calculate coefficients of $a_{i}$ commutatively, when we consider in $\bar{I}_{G}$ modulo $\bar{I}_{H} I_{M}$. And it is shown that

$$
\bar{I}_{H} I_{M} \cap I_{G}^{H \rightarrow 0} \subset I_{H} I_{G}
$$

Now let $a=\sum_{i=1}^{m} \gamma_{i} a_{i} \in I_{G}$. Then

$$
\begin{aligned}
S^{*} a & =S^{*} \sum \gamma_{i} a_{i} \equiv \sum \gamma_{i} \cdot S^{*} a_{i}=\sum \gamma_{i}\left(1-\tau_{i}^{-1} \bar{S}^{-1} \tau_{i} \bar{S}\right) \\
& =\sum \gamma_{i} \tau_{i}^{-1} \bar{S}^{-1}\left((1-\bar{S}) a_{i}-\left(1-\tau_{i}\right) a_{0}\right) \quad \bmod \left(I_{G^{\prime}}, I_{H} I_{G}\right) .
\end{aligned}
$$


On the other hand $r a=\sum_{\sigma_{i} \in G / H} \sigma_{i} \cdot \sum_{i=1}^{m} \gamma_{i} a_{i^{*}} \quad$ Let $f_{i}=1+\tau_{i}+\cdots+\tau_{i}^{e^{i}}{ }^{-1}$. Then, $\tau_{i}^{-1} f_{1} \cdots f_{m} a_{i} \equiv f_{1} \cdots f_{m} a_{i}\left(\bmod I_{H} \cdot I_{G}\right)$, and

$$
\begin{aligned}
\bar{S}^{-1}(r \cdot a) & =\bar{S}^{-1} f_{1} \cdots f_{m} \sum \gamma_{i} a_{i} \equiv \bar{S}^{-1} \sum \gamma_{i} f_{1} \cdots f_{m} a_{i} \\
& \equiv \sum \gamma_{i} \tau_{i}^{-1} \bar{S}^{-1} f_{1} \cdots f_{m} a_{i} .
\end{aligned}
$$

Let $\eta_{i}=\gamma_{i} \tau_{i}^{-1} \bar{S}^{-1}$, then our proposition is reduced to the following:

"If $\sum \eta_{i}\left((1-\bar{S}) a_{i}-\left(1-\tau_{i}\right) a_{0}\right) \in\left(\bar{I}_{G^{\prime}}, \bar{I}_{H} I_{M}\right)$, then

$$
\sum \eta_{i} f_{1} \ldots f_{m} a_{i} \in \bar{I}_{H} I_{M} " . \quad \text { (cf. (3)) }
$$

Proof OF THE PROPOSITION. As $\tau_{i}^{e} \in H, 1-\tau_{i}^{e} \in \in I_{H}$ is expressed by (1), (2) as follows.

$$
1-\tau_{i}^{e_{i}}=\sum_{k>l}^{1, \cdots, m} P_{k l}^{(i)}\left(\left(1-\tau_{l}\right) a_{k}-\left(1-\tau_{k}\right) a_{l}\right)+\sum_{k=1}^{m} P_{k 0}^{(i)}\left((1-S) a_{k}-\left(1-\tau_{i}\right) a_{0}\right) .
$$

In this formula, $1-\tau_{i}^{e} e_{i} f_{i}$; and if we rewrite

$$
\begin{aligned}
& -\sum P_{k l}^{(i)}\left(\left(1-\tau_{l}\right) a_{k}-\left(1-\tau_{k}\right) a_{l}\right)-\sum P_{k 0}^{(i)}(1-\bar{S}) a_{k}=\sum Q_{k}^{(i)} a_{k} \\
& -\sum P_{k 0}^{(i)}\left(1-\tau_{k}\right) a_{0}=R_{i} a_{0},
\end{aligned}
$$

we have

$$
f_{i} a_{i}+\sum_{k=1}^{m} Q_{k}^{(i)} a_{k}=R_{i} a_{0} \quad(i=1, \cdots, m)
$$

By the elimination formula, we have

$$
D_{l} a_{k}=D_{k} a_{l} \quad(k, l=0,1, \cdots, m)
$$

where

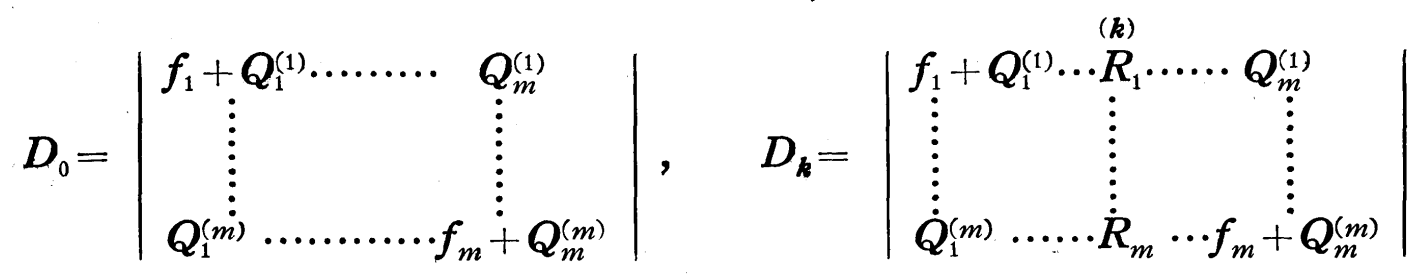

Since we can calculate the coefficients of $a_{k}$ commutatively, we 
have from (4),

$$
\sum Q_{k}^{(i)}\left(1-\tau_{k}\right) a_{l}=-\sum P_{k 0}^{(i)}(1-\bar{S})\left(1-\tau_{k}\right) a_{l}=(1-\bar{S}) R_{i} a_{0} .
$$

Also we have $\left(1-\tau_{k}\right) f_{k} a_{l}=0$. Therefore, after multiplying the first row of $D_{0}$ by $1-\tau_{1}, \cdots$, the last row of $D_{0}$ by $1-\tau_{m}$, we have the following formula by adding each row to the $k$-th row:

$$
\left(1-\tau_{k}\right) D_{0} a_{l}=(1-\bar{S}) D_{k} a_{l} \quad(l=0,1, \cdots, m ; \quad k=1, \cdots, m)
$$

Thus we get elements $D_{k}$ of $M$, which satisfy (6) and (7). Since $(1-\bar{S})\left(1+\bar{S}+\cdots+\bar{S}^{l-1}\right)=0$, we may assume that the $\bar{S}$-degree of $D_{k}\left(k=1, \cdots, m\right.$; that is, except $\left.D_{0}\right)$ is at most $l-2$.

Since $M$ is the direct sum of $Z(G)$ and $M(1-\bar{S}), D_{0}$ can be described as $D_{0}=D^{\prime}+D(1-\bar{S})$, where $D^{\prime} \in Z(G)$ and the $\bar{S}$-degree of $D$ is at most $l-2$. Therefore, we have from (7)

$$
\left(1-\tau_{k}\right) D^{\prime} a_{l}=(1-\bar{S})\left(D_{k}-\left(1-\tau_{k}\right) D\right) a_{l}
$$

Since the left-hand side of this equality is an element of $Z(G)$, and the $\bar{S}$-degree of $D_{k}-\left(1-\tau_{k}\right) D$ is at most $l-2$, we have the following two relations.

$$
\begin{aligned}
& D_{k} a_{l}=\left(1-\tau_{k}\right) D a_{l} \quad(l=0,1, \cdots, m ; k=1, \cdots, m) \\
& \left(1-\tau_{k}\right) D^{\prime} a_{l}=0
\end{aligned}
$$

From the second formula, we have $D^{\prime} a_{l}=f_{1} \cdots f_{m} D^{\prime \prime} a_{l}$, and then

$$
D_{0} a_{l}=D(1-\bar{S}) a_{l}-f_{1} \cdots f_{m} D^{\prime \prime} a_{l} \quad(l=1, \cdots, m)
$$

When we consider this relation modulo $I_{M} I_{M}$, we have $e_{1} \cdots e_{m} a_{l}=$ $e_{1} \cdots e_{m} D^{\prime \prime}(1) a_{l}$, and this implies $D^{\prime \prime}(1)=1$. Since $D^{\prime \prime} \in Z(G), D^{\prime \prime}(1)=1$ means $f_{1} \cdots f_{m} D^{\prime \prime} a_{l}=f_{1} \cdots f_{m} a_{l}$. Therefore, we get the following relation

$$
D_{0} a_{l}=D(1-\bar{S}) a_{l}-f_{1} \cdots f_{m} a_{l} \quad(l=1, \cdots, m)
$$

Thus we have an element $D$ which satisfies (8) and (9).

Now let us compute $\sum \eta_{i} f_{1} \cdots f_{m} a_{i}$. It is performed by (6) (9). 
Let $\sum_{i=1}^{m} \eta_{i} a_{i}$ be an element of $I_{G}$, which satisfies

$$
\sum \eta_{i}\left((1-\bar{S}) a_{i}-\left(1-\tau_{i}\right) a_{0}\right)=\sum_{i, j=1}^{m} f_{i j}\left(\left(1-\tau_{i}\right) a_{j}-\left(1-\tau_{j}\right) a_{i}\right)
$$

Then

$$
\begin{aligned}
\sum \eta_{i} f_{1} \cdots f_{m} a_{i} & =\sum \eta_{i}\left(D_{0} a_{i}-D(1-\bar{S}) a_{i}\right), & & \text { by }(9) \\
& =\sum \eta_{i}\left(D\left(1-\tau_{i}\right) a_{0}-D(1-\bar{S}) a_{i}\right), & & \text { by }(6) \text { and }(8) \\
& =D \cdot \sum f_{i j}\left(\left(1-\tau_{j}\right) a_{i}-\left(1-\tau_{i}\right) a_{j}\right), & & \text { by the assumption } \\
& =0 & & \text { by }(6),
\end{aligned}
$$

which proves our proposition.

Since $\psi$ and $\varphi$ are isomorphisms, our proposition implies that the kernel of $S_{2}^{*}$ is contained in the kernel of $\boldsymbol{r}_{2}$.

4. Let $\bar{\Omega} / \Omega$ be a finite normal extention of an algebraic number field $\Omega, G$ be the Galois group of $\bar{\Omega} / \Omega$, and $S$ be an automorphism of the group $G$.

Let $H$ be the invariant subgroup of $G$ which is considered in 1., $K$ be the corresponding intermediate field of $\bar{\Omega} / \Omega$.

Let $C_{\bar{\Omega}}, C_{K}$, and $C_{\Omega}$ be the idele class groups of $\bar{\Omega}, K$, and $\Omega$, respectively. Let $\xi$ be the canonical class of $H^{2}\left(G, C_{\bar{\Omega}}\right)$. Then $\zeta \rightarrow$ $\xi \otimes \zeta\left(\zeta \in H^{-2}(G, Z)\right)$ induces an isomorphism of $H^{-2}(G, Z)$ with $H^{0}(G$, $\left.C_{\bar{\Omega}}\right)$. And, combining with this isomorphism, $S_{2}^{*}$ induces a homomorphism $S_{0}^{*}$ of $H^{0}\left(G, C_{\bar{\Omega}}\right)$ into $H^{0}\left(H, C_{\bar{\Omega}}\right) /\left(\right.$ kernel of $\left.j_{0}\right)$. Then, $j_{0} S_{0}^{*}=S^{*}$ defines an endomorphism of the class group $C_{\Omega} / N_{\bar{\Omega} / \Omega} C_{\bar{\Omega}}$.

On the other hand, the restriction mapping $r_{0}$ of the group $H^{0}\left(G, C_{\bar{\Omega}}\right)$ into $H^{0}\left(H, C_{\bar{\Omega}}\right)$ induces the injection mapping of $C_{\Omega} / N_{\bar{\Omega} / \Omega} C_{\bar{\Omega}}$ into $C_{K} / N_{\bar{\Omega} / K} C_{\bar{\Omega}}$. Therefore, we have the following theorem from the preceding proposition.

THEOREM. The kernel of the endomorphism $j_{0} S_{0}^{*}$ of the group $C_{\Omega} / N_{\bar{\Omega} / \Omega} C_{\bar{\Omega}}$ is contained in $N_{\bar{\Omega} / K} C_{\bar{\Omega}}$, when it is considered in $C_{K}$.

A special case of this theorem is the generalised principal ideal theorem which was obtained by Prof. Tannaka and the author. Now, let $k$ be a finite algebraic number field and $K$ be the absolute class 
field (generally a ray class field) over $k$. Let $\Omega / k$ be a cyclic intermediate field of $K / k$, and $S$ be a generator of the (cyclic) Galois group of $\Omega / k$. Let $\bar{\Omega} / K$ be the absolute class field of $K$, and $G$ be the Galois group of $\bar{\Omega} / \Omega$. Then $S$ induces an automorphism of the group $G$, and $K$ is just the intermediate field of $\bar{\Omega} / \Omega$ which is considered in the preceding theorem. It is easy to see that the homomorphism $j_{0} S_{0}^{*}$ in the theorem is the endomorphism such that $c \rightarrow c^{1-s}$, where $c \in C_{\ell}$ and $c^{s}$ means the image of $c$ by the element $S$ of the Galois group of $\Omega / k$. Thus we have the following theorem.

THEOREM. Let $k$ be an algebraic number field, $K$ the absolute class field over $k$, and $\Omega / k$ a cyclic intermediate field of $K / k$. Let $S$ be a generator of the Galois group of the cyclic extension $\Omega / k$. Then all ambigous classes in $\Omega$ (i.e. idele class $c$ such that $c^{1-s}=1$ ) are contained in $N_{\bar{Q} / K} C_{\bar{\Omega}}$, when considered in $K$.

By the usual correspondence which exists between ideles and ideals we have an analogous result which was obtained in [2] and [3]. The description of our theorem by an automorphism is due to a suggestion by K. Masuda.

\section{Tōhoku University}

\section{References}

[1] C. Chevalley, Class field theory, Nagoya University, 1953-1954.

[2] T. Tannaka, Some remarks concerning principal ideal theorem, Tōhoku Math. Journal, second series, vol. 1 (1951).

[3] F. Terada, On generalized principal ideal theorem, Tohoku Math. Journal, second series, vol. 6 (1954), 95-100. 